\title{
Wetting of crystalline polymer surfaces: A molecular dynamics simulation
}

\author{
Cun Feng Fan ${ }^{\mathrm{a}, \mathrm{b})}$ and Tahir Cağin ${ }^{\mathrm{c})}$ \\ Molecular Simulations Inc., Pasadena Advanced Research Center, 600 South Lake Avenue, Suite 411, \\ Pasadena, California 91101
}

(Received 2 May 1995; accepted 10 August 1995)

\begin{abstract}
Molecular dynamics has been used to study the wetting of model polymer surfaces, the crystal surfaces of polyethylene (PE), poly(tetrafluoroethylene) (PTFE), and poly(ethylene terephthalate) (PET) by water and methylene iodide. In the simulation a liquid droplet is placed on a model surface and constant temperature, rigid body molecular dynamics is carried out while the model surface is kept fixed. A generally defined microscopic contact angle between a liquid droplet and a solid surface is quantitatively calculated from the volume of the droplet and the interfacial area between the droplet and the surface. The simulation results agree with the trend in experimental data for both water and methylene iodide. The shape of the droplets on the surface is analyzed and no obvious anisotropy of the droplets is seen in the surface plane, even though the crystal surfaces are highly oriented. The surface free energies of the model polymer surfaces are estimated from their contact angles with the two different liquid droplets. () 1995 American Institute of Physics.
\end{abstract}

\section{INTRODUCTION}

The determination of wetting properties of a polymer surface is extremely important in many of its technological applications. Over the last few decades, considerable progress has been made in understanding the wetting phenomenon both in theoretical and experimental fronts. ${ }^{1-4}$ The wetting of a surface is essentially determined by the molecular interactions between the liquid and the surface. The prediction of wettability for a particular liquid/surface combination inevitably requires detailed information regarding their chemical composition, the surface structure and geometry, and the dynamics of the liquid and surface interface. Such information is inherently contained in atomistic simulation of materials. Hence, the molecular dynamics simulation of polymer surface and liquid interface is capable of exploring the nature of surface wetting.

The concept of contact angle is frequently used to characterize the degree of wetting of a surface of a solid by a liquid droplet. The contact angle, a macroscopic parameter, can be measured accurately from experiments. Using the contact angle information one may derive the surface free energy of a solid surface. However, the conventional contact angle becomes ill-defined at the molecular level, as depicted in Fig. 1. Apparently, a more general interpretation of the contact angle for a microscopic configuration of a droplet should be found so that the quantitative comparison between the results obtained from molecular simulations and the experiments can be made.

The wetting of a surface is essentially characterized by the shape of the liquid droplet on it. One can relate the shape to the contact angle. The two extreme shapes are a sphere and a planar film. Everything else in between may be thought to the crown of a sphere. Then, the contact angle may unam-

\footnotetext{
a) To whom correspondence should be addressed.

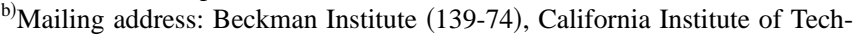
nology, Pasadena, CA 91125.

${ }^{c}$ Current address: Beckman Institute (139-74), California Institute of Technology, Pasadena, CA 91125.
}

biguously be defined from some geometric parameters of a droplet. Consequently, this definition can be used in computing the contact angle for an irregular droplet for which such geometric parameters are obtainable.

In a recent molecular dynamics study of the wetting of hydrophobic and hydrophilic surfaces by water, Hautman and Klein calculated microscopic wetting angle along these lines. They established a quantitative correlation between the macroscopic contact angle and microscopic structure of droplet through a single geometric parameter. ${ }^{5}$ With such a relation established, they were able to make a realistic prediction of surface wetting from the molecular structures of liquid and surface. The method is elegant and the results compared favorably with experimental data. ${ }^{5,6}$ In order to extend the molecular level simulation approach to a broader set of applications, some of the assumptions used in this method need to be considered. In the above mentioned calculation two assumptions are made. First assumption is on the density of water droplet. It is taken as the experimental density, the density fluctuations are not taken into account in the course of simulation and density is taken to be uniform through the extent of the droplet. Secondly, in the calculation, a planar surface was defined. The height of this plane is defined as the average heights of surface atoms. The center of mass of a droplet was then calculated by using the planar surface as the reference. With these two assumptions it was possible to derive the contact angle from a single microscopic parameter, namely the height of center of mass of the droplet from the surface.

In this paper, we adopt an alternative approach to calculate the instantaneous contact angle from the microscopic structures of the droplet and the surface. The instantaneous contact angle expression is derived from the volume of the droplet and interfacial area between the droplet and the surface, both are calculated from the molecular dynamics trajectories. No a priori assumption is made for the density of droplet. The planar surface assumption is more or less arbitrary and may not be generally applicable, especially for rough surfaces. In this approach, the solid surface is defined 
(a)

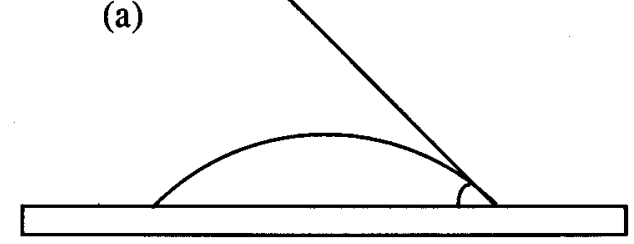

(b)

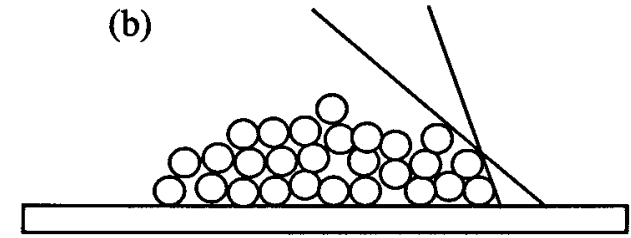

FIG. 1. Schematic drawing of macroscopic and microscopic droplets. The conventional contact angle for microscopic droplet becomes ill-defined.

from the van der Waals profile of the model polymer surface. This practically enables us to apply the method to any surface geometry including the rough surfaces. The method is then applied to several model crystal polymer surfaces. The surface free energies of these model surfaces are also estimated.

The layout of the paper is as follows. In the next section, we will describe the model systems and simulation techniques used. In Sec. III we present the method and the algorithms employed in determining the contact angles. In Sec. IV, we present and discuss the results of application to model polymer surfaces.

\section{MODELS AND SIMULATION TECHNIQUES}

The polymers studied are polyethylene (PE), poly(tetrafluoroethylene) (PTFE), and poly(ethylene terephthalate) (PET). The contact angles of these polymers with water encompass a broad range, therefore, the models can serve as good indicators of the sensitivity of the method developed here. The model surfaces are built from the crystal structures of the polymers with the experimental unit cell parameters. ${ }^{7}$ The orthorhombic structure (Pnam- $D_{2 h}{ }^{16}$ ) is used to build PE surface. For PTFE we used the trigonal form with the backbone dihedral angle $=165.8^{\circ}(15 / 7$ helix $)$, since it is the stable structure above $19{ }^{\circ} \mathrm{C}$. PET has only one form, triclinic $\left(\mathrm{P} \overline{1}-\mathrm{C}_{i}^{1}\right)$. The (100) surfaces of the crystal lattice are used as the surface planes in all cases. Most of the real polymer surfaces are amorphous, even the bulk polymer are semicrystalline. However, highly crystalline surfaces can be achieved under certain conditions. ${ }^{3}$ The main objective of this study is to perform quantitative calculation of contact angle from microscopic structure. By choosing well defined crystalline surfaces, any ambiguity which might have arisen from the surface structure itself is removed. The structure of polymer surface, being amorphous or semicrystalline, can be very complex and the subject is a very active area on its own. ${ }^{8}$ The method developed here can be applied to a less idealized surface if a good representation of a realistic surface can be generated in the modeling. The dimensions of the model surfaces are about $100 \AA \times 100 \AA$ with a thickness of $\sim 10 \AA$. The model surfaces were fixed during the molecular dynamics simulation. The force field for the polymers is Dreiding ${ }^{9}$ with partial charges calculated from the Gasteiger method. ${ }^{10}$

The water droplet contains 216 water molecules. The extended simple point charge model $(\mathrm{SPC} / \mathrm{E})^{11}$ was used. It differs from the original simple point charge model (SPC) ${ }^{12}$ with $q_{\mathrm{O}}=-0.8476 e$ (the partial charge on oxygen) instead of $-0.82 e$ in the SPC model where $e$ is the electron charge unit. The charge on the hydrogen atom $\left(q_{H}\right)$ equals $-0.5 q_{\mathrm{O}}$. The original SPC model is quite satisfactory to produce liquid water structure. ${ }^{13}$ It has been used to study the liquid-vapor coexistence curve ${ }^{14}$ and the surface wetting. ${ }^{5}$ However, more recent studies showed that the SPC/E model performed better in the study of liquid-vapor equilibrium and estimation of surface tension. ${ }^{15,16}$ In order to estimate the surface free energy of the model polymer surfaces, methylene iodide $\left(\mathrm{CH}_{2} \mathrm{I}_{2}\right)$ is chosen as the second liquid droplet (256 molecules) in addition to water. The interaction parameters used for the liquid methylene iodide are also from the Dreiding force fields with the partial charges calculated using the charge equilibration method. ${ }^{17}$ All the liquid molecules are treated as rigid bodies (no internal degrees of freedom). Force field representations for the liquids were checked by MD simulations of both liquids without surface. In these simulations both of the liquids formed stable droplets.

The initial configuration of liquid droplet is generated in two steps. First, a MD simulation with periodic boundary conditions (PBC) is performed to obtain correct bulk structure. Then, additional MD simulation without $\mathrm{PBC}$ is run to obtain an equilibrated isolated droplet. In the subsequent simulation of surface wetting such droplet is placed on a model polymer surface. The constant temperature simulations (using Nose thermostat ${ }^{18}$ ) are performed at $300 \mathrm{~K}$ for the droplet while the model surface is kept fixed. Rigid body constraints are imposed by employing the rigid body quaternion algorithm. ${ }^{19}$ This enabled us to use the integration time steps of $0.005 \mathrm{ps}$ for water and $0.01 \mathrm{ps}$ for $\mathrm{CH}_{2} \mathrm{I}_{2}$. First, a $150 \mathrm{ps}$ simulation (water) is run to reach the equilibrium contact angles. Subsequently, additional 100 ps runs, starting with the structures extracted from equilibration runs, are performed to collect the data for the equilibrium contact angle calculation. The equilibrium contact angle is independent of the initial configurations of the droplet, therefore, it corresponds to the static (thermodynamics) contact angle. It should lie between the advancing contact angle and receding contact angle obtained in the dynamic contact angle measurements. ${ }^{4}$ The differences between the static and dynamic contact angles will decrease as the surface becomes smoother, and both the surface and the liquid become purer. ${ }^{4}$ The equilibration time for $\mathrm{CH}_{2} \mathrm{I}_{2}$ is significantly longer (on the order of $300 \mathrm{ps}$ ). The structures are saved every $0.1 \mathrm{ps}$. The nonbonded interactions between the droplet and the surface are calculated from the entire surface and the whole droplet. No external pressure or reflective wall is used in the simulation. The vapor density is very low at $300 \mathrm{~K}$ as shown in the study of liquid-vapor equilibrium of water. ${ }^{16}$ In the course of a simulation mostly none, or on occasion one or 

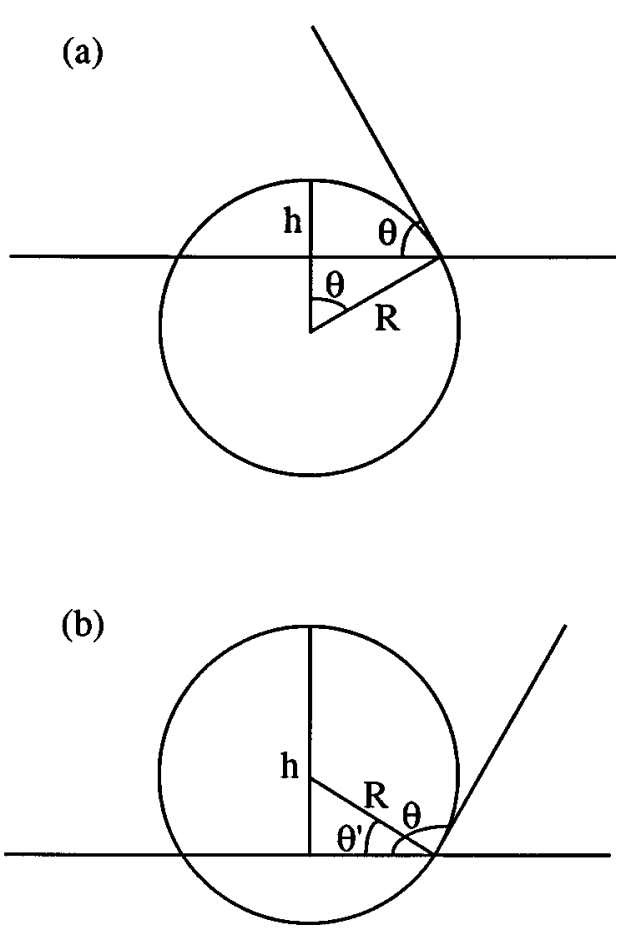

FIG. 2. Geometry for defining contact angle for intersected spheres.

two of the liquid molecules escaped (evaporated) from the droplet.

\section{CALCULATION OF CONTACT ANGLE}

\section{A. Definition of contact angle}

The instantaneous contact angle is calculated for each frame of the MD trajectories. As in the approach presented by Hautman and Klein ${ }^{5}$ (see Appendix) the contact angle of irregular microscopic droplet is calculated from well defined geometric parameters, which are generally applicable for both intersected sphere and irregular shape droplet. A general relationship between the parameters and the contact angle of an intersected sphere is derived. Here, we outline the method used in our calculation. Figure 2, shows two droplets (one with $\theta<90^{\circ}$ and the other one with $\theta>90^{\circ}$ ) on a surface. Here $\theta$ is the contact angle, $h$ is the height of the droplet, and $R$ is the radius of the sphere. In the case of $\theta<90, \cos \theta=(R$ $-h) / R$. Similarly for $\theta>90$, one obtains $\cos \theta=-\sin \theta^{\prime}$ $=-(h-R) / R$. The contact angle can be defined as

$$
\cos \theta=1-\frac{h}{R} .
$$

The $h$ and $R$ in the equation can be calculated from the volume of the droplet $V$ with $V=\pi h\left(3 r^{2}+h^{2}\right) / 6$ and interfacial area $S$ with $S=\pi r^{2}$. Here $r$ is the radius of the intersection circle as depicted in Fig. 3. Since $(R-h)^{2}+r^{2}=R^{2}$ the following pair of equations are obtained:

$$
h^{3}+\frac{3 S}{\pi} h-\frac{6 V}{\pi}=0
$$

and

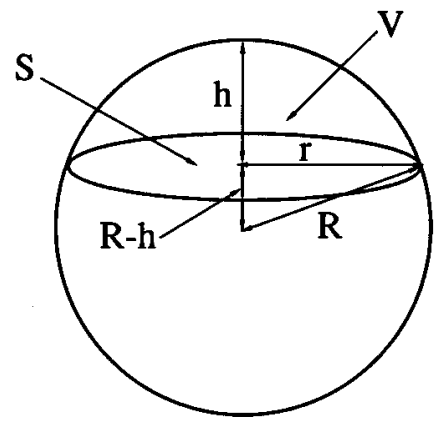

FIG. 3. Geometry for calculating droplet volume and interfacial area.

$$
R=\frac{h}{2}+\frac{S}{2 \pi h} .
$$

Only the real root of the third order equation [Eq. (2)] is meaningful. Although, they are derived from the same intersected sphere construction of Ref. 5, Eqs. (1)-(3) together represent a more general definition of contact angle. Two parameters of these equations, volume $(V)$ and interfacial area $(S)$, may be calculated directly from the simulation for each configuration and are applicable to droplets of any shape. Using the above prescription, the degree of surface wetting for any microscopic configuration of a droplet can be quantitatively measured and compared with macroscopic experimental data.

\section{B. Calculation of the volume of a droplet}

To calculate the volume, $V$, a three dimensional grid is imposed on simulated liquid droplet. Occupied and unoccupied sites for this grid are determined as follows. A site in the volume grid is counted as occupied if it is within the van der Waals radius of any atom. The total volume of the droplet, $V$, is calculated as the sum of all the occupied sites and the interior unoccupied sites (the sites which are entrapped by the occupied sites). An interior unoccupied site is defined as the one with no straight channel connecting it to outside of droplet. Traveling along any of the six directions $( \pm x, \pm y$, and $\pm z$ ) from an interior unoccupied site, it will always meet an occupied site (see Fig. 4). If any of the six directions is not being blocked by an occupied site, this unoccupied site contribution to volume is not included. The interior unoccupied sites may be considered as the free volume portion of a droplet. Such calculation of the volume of a droplet is physically sound and the density obtained agrees with experimental value.

\section{Calculation of the interfacial area}

First, the van der Waals profile of a surface is calculated with the normal vector to the surface being parallel to $z$ axis. For each atom in the model surface, a projection circle on a two dimensional surface grid is constructed. Each grid site within the circle is assigned to a height $z_{s}=z_{0}+z_{1}$ where $z_{0}$ is the $z$ coordinate of the atom and $z_{1}=d L^{*} L_{3}$, where $d L$ is the grid resolution $(0.4 \AA)$ and $L_{3}$ is obtained from $L_{3}^{2}=R^{2}-L_{1}^{2}-L_{2}^{2}$. Here $L_{i}(i=1,2,3)$ is the site position 


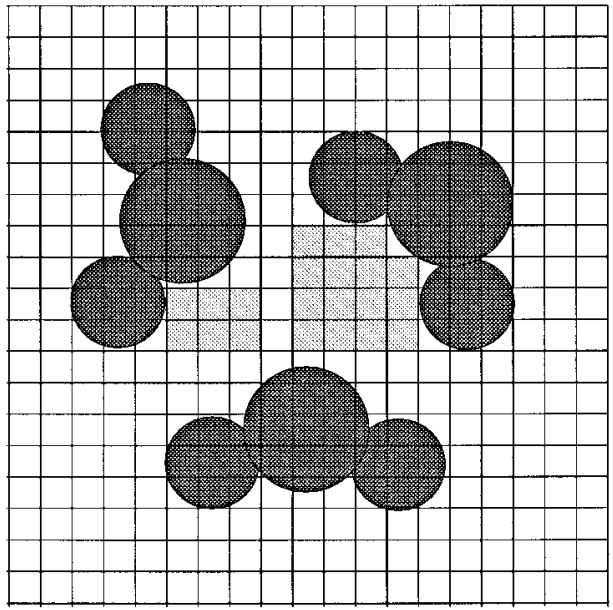

FIG. 4. A slice of the three dimensional mesh for the calculation of the volume of a droplet. Darker shadow: occupied sites; lighter shadow: interior empty sites; white: empty sites.

relative the center of atom, $R$ is the van der Waals radius of the atom. This process is repeated for all of the atoms in the surface and the maximum height of each site in the surface grid is updated. The final maximum heights of all the sites in the two dimensional grid represent the surface van der Waals profile.

To calculate the interfacial area, we first determine whether or not an atom in a droplet is in contact with the surface. A similar projection circle for each atom in the droplet is made on the same two dimensional grid. Each grid site within this van der Waals circle is assigned a height $z_{d}=z_{0}-z_{1}$ here $z_{0}$ is the $z$ coordinate of the atom in the droplet and $z_{1}$ has the same meaning as in the previous paragraph. If any site within the circle satisfies $z_{d} \leqslant z_{s}$ the atom is considered in contact with the surface and all the grid sites within the projection circle are assigned to be in contact with the surface (see Fig. 5). The total interfacial area is the sum of all the grid areas which are in contact with the surface. This approach generates detailed microscopic information about the wetting process. For example, the isolated area surrounded by the wetted surface can be quantitatively measured and visualized. Clearly, the method is generally applicable to a system with any surface geometry and roughness.

\section{RESULTS AND DISCUSSION}

\section{A. The wetting process and contact angle}

The initial height of a droplet on a surface is determined such that no severe overlaps between the droplet and surface are allowed. Such positioning, however, only allows few atoms in the droplet to be in contact with the surface. As the simulation proceeds, the molecules in the droplet are attracted to the surface and spread to a larger area. Such process can be visualized in Fig. 6 while the contact surface at several different time frames for the water/PET system is shown. Figure 7 plots the variation of volume of the droplet and contact area as a function of time for the same system. As expected, the contact area initially increases and then reaches to steady state. For the water droplet the equilibra-

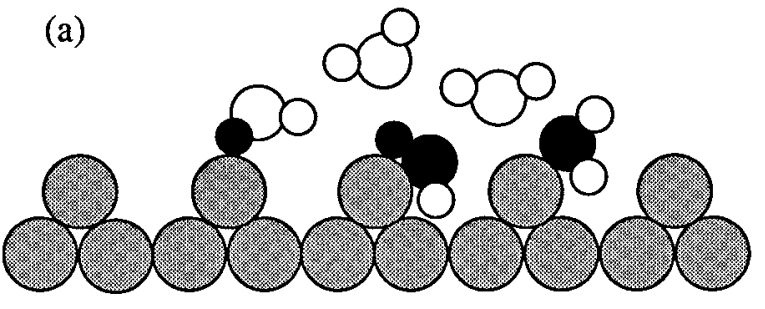

(b)

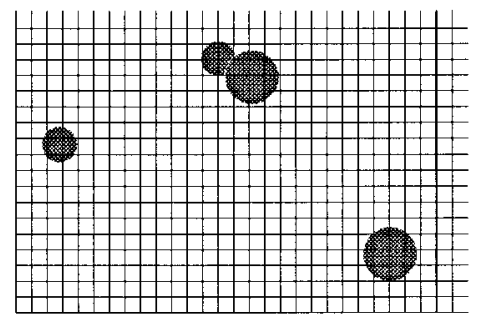

FIG. 5. Calculation of interfacial area between a droplet and a surface. (a) atoms (black) in contact with the surface (gray); (b) the interfacial areas (shadow) due to these contacts as projected on a two dimensional grid.

tion time is less than $50 \mathrm{ps}$. All three water/polymer systems display similar behavior, except the equilibrium contact angles are different. The volume of the droplet fluctuates during the simulation. The averaged volume of a droplet on the surface is the same as the one of an isolated water droplet in the absence of the surface.

Two microscopic configurations of water droplet on PET surface at different time frames are shown in Fig. 8. They represent the snapshots in the early stage $(7.5 \mathrm{ps})$ and late stage $(136.7 \mathrm{ps})$ of wetting, respectively. The contact angle calculated from the volume and the interfacial area of water/ PET system is plotted in Fig. 9. Similar to the plot seen in Fig. 7, the equilibrium time for water droplet is less than 50 ps, almost regardless of the model surface. The equilibrium time for $\mathrm{CH}_{2} \mathrm{I}_{2}$ is considerably longer, about 300 ps for the same system. The long equilibration time for methylene iodide is due to the difference in the inertia of the molecules and the equilibrium angles they attain. The contact angles of $\mathrm{CH}_{2} \mathrm{I}_{2}$ are significantly smaller than water for all the model surfaces studied here. Table I lists the time averaged contact angles obtained from the simulation along with the experimental data. ${ }^{20}$ However, one should be very cautious to make such comparison due to the fact that the real polymer surfaces are more likely to be amorphous while perfect crystalline model surfaces are employed in the simulation. Normally, the crystalline surface has lower contact angle. In principle the difference between the simulated contact angle and corresponding literature value can be traced to two sources: the difference between the model system and the real system, even if both were amorphous, and the additional difference between the crystalline surface and the amorphous surface. Another fact should be considered as well: the surfaces are fixed in the simulation. The structural changes induced by the droplets are assumed to be minimal for the perfect crystalline surfaces studied here. In general such mu- 

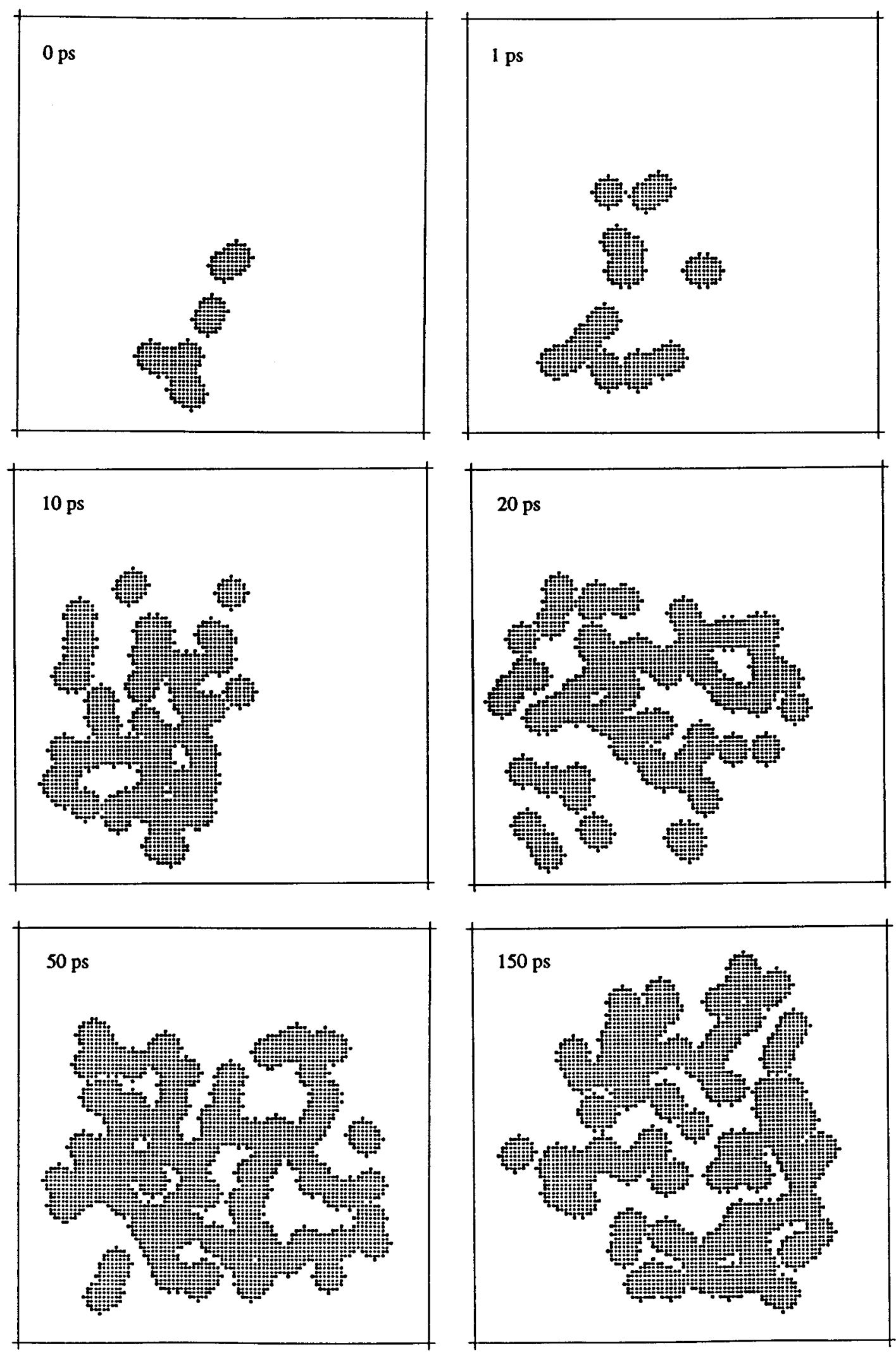

FIG. 6. Selected frames of the interfacial area of water PET at different time.

tual interactions can be important for rough surfaces and surfaces with impurities. The results obtained from the simulation qualitatively agree with the experimental data and reproduce the trend observed in the experiments. The larger contact angles from simulation were observed previously ${ }^{5}$ and this may well be a general phenomena. The ideal models used in the simulations are highly purified (without any impurity which always exists in real surfaces). The impurities 


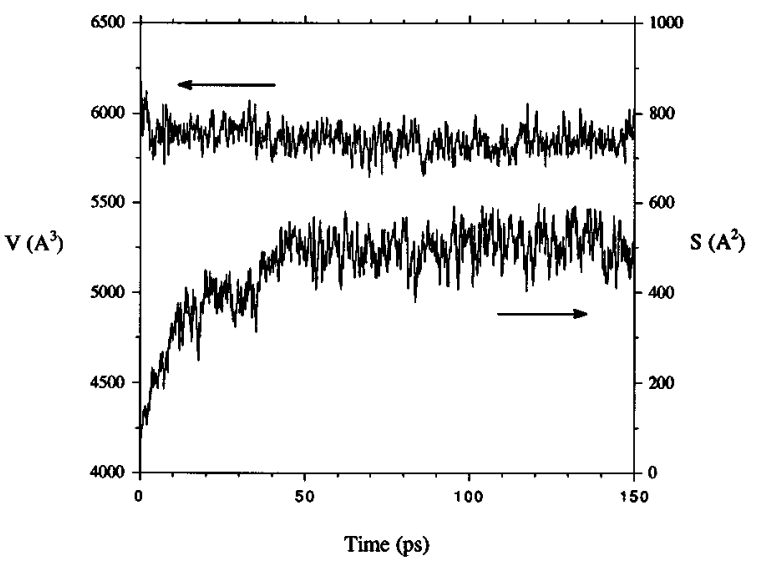

FIG. 7. The volume of the water droplet and its interfacial area with PET as a function of time.

normally tend to enhance the surface wetting. The main differences between the three model surfaces are their chemical compositions and chain packings. Our results clearly indicate that the chemical compositions of the surface play a major role in wetting process between the droplet and the surface. The effect of chain packing (i.e., crystal structure) is not fully explored yet.

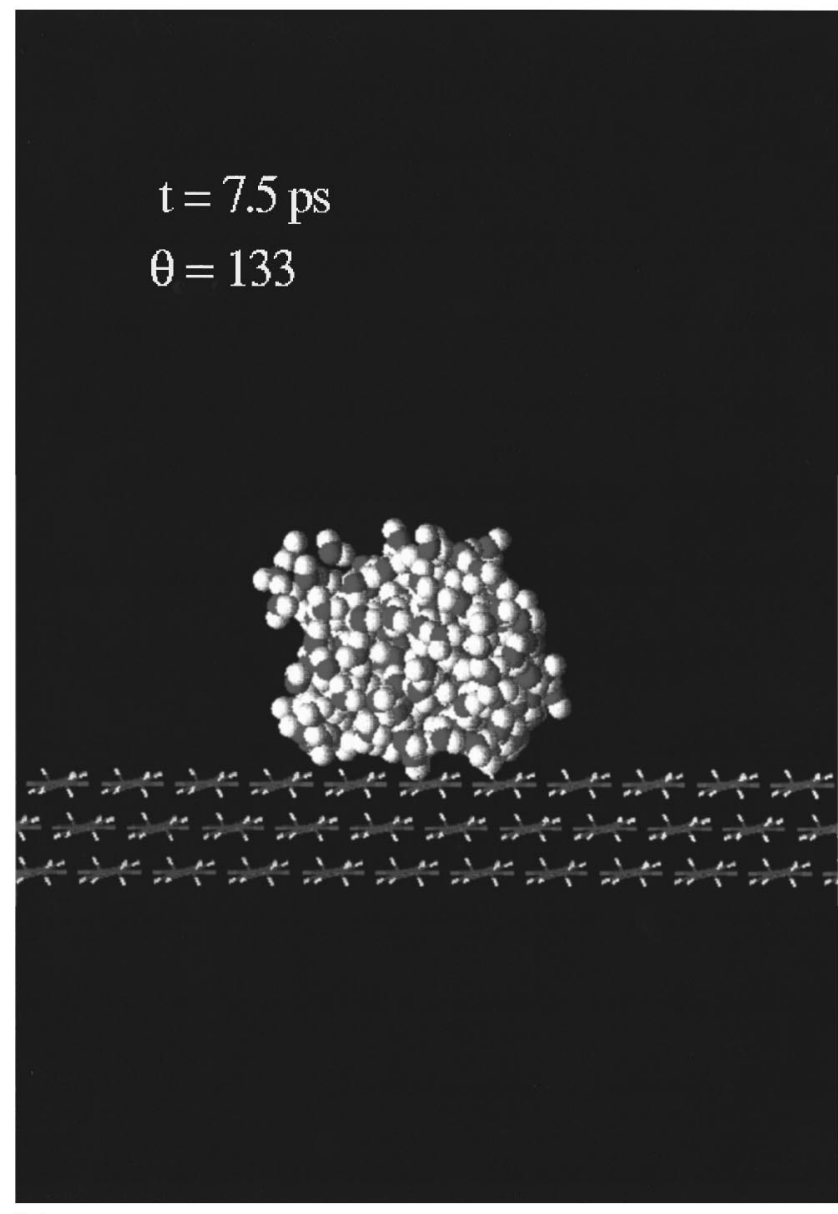

(a)

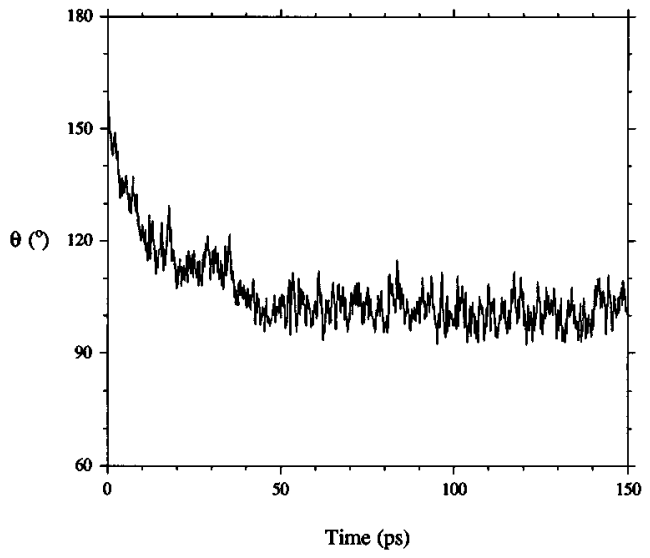

FIG. 9. Contact angle between water droplet and model PET surface during the equilibration segment of the simulation.

\section{B. Estimation of surface free energy}

The surface free energy (surface tension) of a polymer can be directly measured through stretching of thin threads, direct measurement of the force between two solids, or extrapolation from melt measurement. ${ }^{2}$ More practical estimation of the surface tension of a polymer involves the measurement of its contact angle with liquid droplets whose

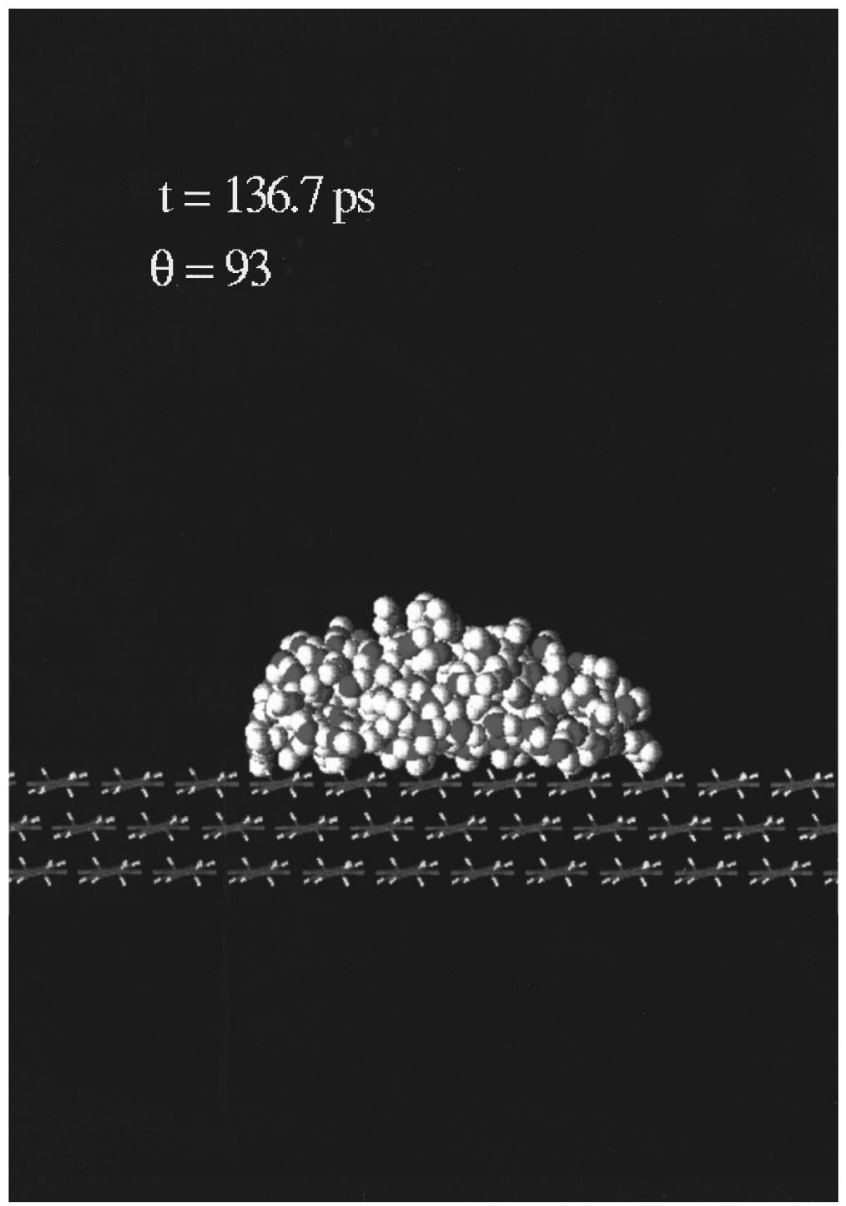

(b)

FIG. 8. Snapshots of water droplet on PET surface. (a) In early stage and (b) in late stage of wetting. 
TABLE I. Simulated and experimental contact angles.

\begin{tabular}{lccccc}
\hline \hline & \multicolumn{2}{c}{ Water } & & \multicolumn{2}{c}{$\mathrm{CH}_{2} \mathrm{I}_{2}$} \\
\cline { 2 - 3 } \cline { 5 - 6 } & Calc. & Expt. (Ref. 20) & & Calc. & Expt. (Ref. 20) \\
\hline PTFE & 127 & 108 & 90 & 88 \\
PE & 121 & 94 & & 76 & 52 \\
PET & 102 & 81 & & 53 & 38 \\
\hline \hline
\end{tabular}

surface tensions are known. This type of measurement primarily includes the Zisman method ${ }^{21}$ with a concept of critical surface tension of wetting and a method used by Owens and Wendt ${ }^{20}$ in which the interfacial tension between a solid and a liquid can be expressed as a function of the arithmetical mean and the geometric mean of the two components. The latter only requires the knowledge of surface tension of two liquids, therefore, is used here for estimation of surface free energy of the model polymers. The equation given by Owens and Wendt ${ }^{20}$ is

$$
1+\cos \theta=\frac{2}{\gamma_{1}}\left(\sqrt{\gamma_{s}^{d} \gamma_{l}^{d}}+\sqrt{\gamma_{s}^{h} \gamma_{l}^{h}}\right),
$$

where $\gamma_{s}$ is the surface free energy of solid, $\gamma_{l}$ is the surface free energy of liquid. The superscripts $d$ and $h$ are for the contribution from dispersion interaction and polar interaction (dipole, hydrogen bonding, etc.), respectively $\left(\gamma_{i}=\gamma_{i}^{d}+\gamma_{i}^{h}\right.$, $i=s, l)$. Although the surface tension of water was calculated directly from MD simulation its dispersion and polar components were not determined from the simulation, nor did the values for methylene iodide. Therefore, we use the well accepted literature data for $\gamma_{l}, \gamma_{l}^{d}$, and $\gamma_{l}^{h}$ for water and $\mathrm{CH}_{2} \mathrm{I}_{2}$ (listed in Table II) in our calculation. Equation (4) gives one equation with two unknowns $\left(\gamma_{s}^{d}\right.$ and $\gamma_{s}^{h}$ ) for each contact angle measurement in the simulation. $\gamma_{s}^{d}$ and $\gamma_{s}^{h}$ for a solid are determined from the contact angle measurements with two different liquids using Eq. (4). The calculated surface tension of three model polymers are shown in Table III, together with the experimental data. The van der Waals interactions seem to contribute the most to the surface free energies of the three model surface which agrees with the experimental measurements performed on a broad range of polymers. $^{20}$ The simulation results reproduce the trend seen in experimental data even though they are about $1 / 3$ to $1 / 4$ lower than the experimental values. The difference could be more prominent if they were compared to the perfect crystalline surfaces. However, the literature values of surface tensions are probably overestimated because of the existence of impurities, disorder and roughness in the real samples, unlike the absolute clean surfaces modeled in the simulation. Nev-

TABLE II. Surface free energy (ergs/ $/ \mathrm{cm}^{2}$ ) of water and $\mathrm{CH}_{2} \mathrm{I}_{2}$ at $20{ }^{\circ} \mathrm{C}$ (Ref. 20).

\begin{tabular}{lccc}
\hline \hline & $\gamma_{1}^{d}$ & $\gamma_{1}^{h}$ & $\gamma_{1}$ \\
\hline Water & 21.8 & 51.0 & 72.8 \\
$\mathrm{CH}_{2} \mathrm{I}_{2}$ & 49.5 & 1.3 & 50.8 \\
\hline \hline
\end{tabular}

TABLE III. Simulated and experimental (Ref. 20) surface free energies (dynes/cm).

\begin{tabular}{lcccc}
\hline \hline & $\gamma_{s}^{d}$ & $\gamma_{s}^{h}$ & $\gamma_{s}$ & $\gamma_{s}$ (expt.) \\
\hline PTFE & 13.5 & 0.1 & 13.6 & 18.5 \\
PE & 20.8 & 0.3 & 21.1 & 31 \\
PET & 32.9 & 0.1 & 33.0 & 43 \\
\hline \hline
\end{tabular}

ertheless, our results indicate that the simulation method applied here is sensitive enough to explore the wetting behavior of different surfaces.

\section{Shape of liquid droplets on the surfaces}

It is informative to use the three principal axes (an ellipsoid) of the radius of gyration tensor of a liquid droplet to characterize its shape on the surface even though the droplets are more or less like an intersected sphere after wetting. Before the MD simulation a liquid droplet is almost spherical with the three principal axes of almost equal lengths. As the simulation proceeds the droplet starts to wet the surface and the shape becomes anisotropic. Such behavior for water/PET system is shown in Fig. 10. This is typical for all the systems studied in this work. The shortest principal axis $\left(S_{3}\right)$ is almost parallel to the $z$ axis (with an angle less then 3 degrees for water/PET). The two longer ones nearly lay on the surface $(x-y)$ plane. Such observations are expected. Since these model crystalline surfaces are highly anisotropic, i.e., all the polymer chains are aligned in a particular direction, it would be more interesting to examine the shape of the droplets in the surface plane. Figure 11 shows the projections of the ellipsoids of water droplets onto the surface planes (averaged over $100 \mathrm{ps}$ production runs). The angles between the longest principal axes and the chain direction are indicated in these schematic drawings. The complete data of the averaged relative lengths of the principal axes and their orientations are listed in Table IV. The $y$ axis is the polymer chain direction in all the model systems studied. The droplets on the model surfaces are fairly isotropic. The ratios between the two longer principal axes range from 1:0.81 to 1:0.90. Water droplet on PE surface has the highest anisotropy (1:0.81)

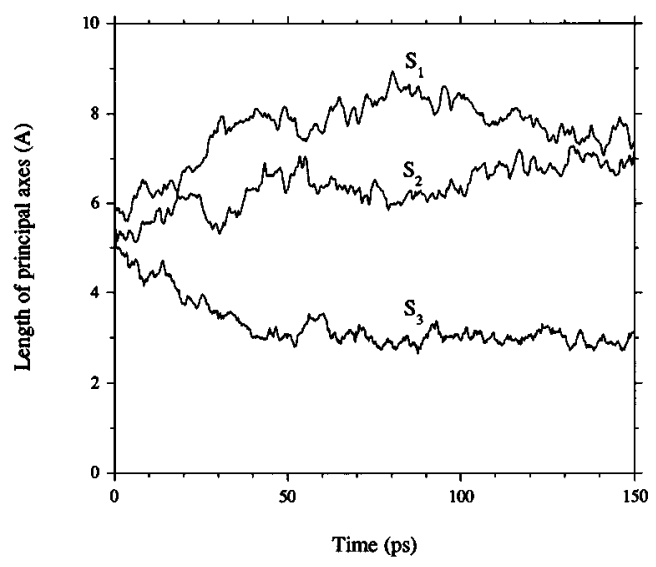

FIG. 10. Principal axes of water droplet on PET surface as a function of time. 
(a)

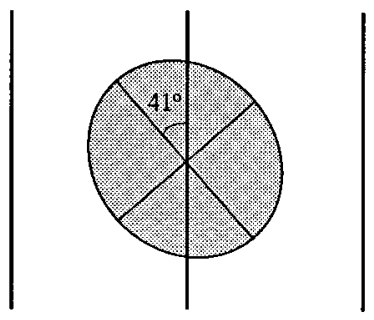

(b)

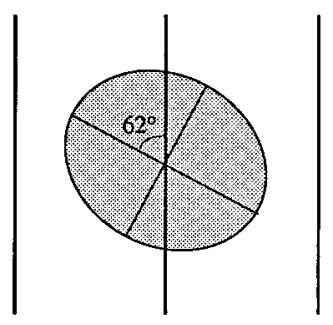

(c)

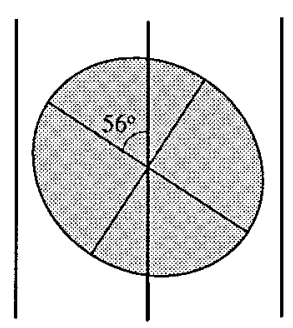

FIG. 11. Projections of radius of gyration ellipsoids of water droplets on the surface planes of (a) PTFE. (b) PE, and (c) PET.

with the longest axis oriented more towards the direction perpendicular to the polymer chains $\left(\sim 62^{\circ}\right.$ with chain direction). The highest orientation angle between the longest axis and chain direction is about $83^{\circ}$ for the $\mathrm{CH}_{2} \mathrm{I}_{2}$ droplet on PET surface, however, such orientation is much less meaningful than the extent indicated by the value since the lengths of the two longer axes are very close. Therefore, the effect of induced orientation by polymer chain on the droplets, either parallel or perpendicular to the chain direction, is insignificant if there is any effect in these systems at all.

\section{CONCLUDING REMARKS}

We have demonstrated that a generally defined microscopic contact angle of a droplet on a solid surface can be

TABLE IV. Relative lengths of principal axes of the droplets and their orientation angles (in degree). $\theta_{S_{i} \hat{k}}$ is the angle between $S_{i}$ and $k$ axis ( $i$ $=1,2,3$ and $k=y, z) ; y$ is parallel to polymer chain direction; $z$ is the surface normal.

\begin{tabular}{lcccccc}
\hline \hline & $S_{1}$ & $S_{2}$ & $S_{3}$ & $\theta_{S_{1} \hat{y}}$ & $\theta_{S_{2} \hat{y}}$ & $\theta_{S_{3} \hat{z}}$ \\
\hline Water & & & & & & \\
PTFE & 1.00 & 0.84 & 0.54 & 40.8 & 49.7 & 5.5 \\
PE & 1.00 & 0.81 & 0.57 & 62.3 & 28.6 & 5.7 \\
PET & 1.00 & 0.88 & 0.40 & 56.4 & 33.4 & 2.9 \\
CH $_{2} I_{2}$ & & & & & & \\
PTFE & 1.00 & 0.90 & 0.33 & 34.0 & 56.1 & 1.7 \\
PE & 1.00 & 0.84 & 0.31 & 35.2 & 54.8 & 1.7 \\
PET & 1.00 & 0.89 & 0.16 & 82.7 & 7.3 & 0.6 \\
\hline \hline
\end{tabular}

determined from the volume of the droplet and the interfacial area between the two. This approach serves as a very useful tool to predict the wetting of a solid and to calculate its surface free energy. The quantitative calculation for the model polymer surfaces from molecular dynamics simulation compares favorably with the experimental measurements, suggesting that the chemical compositions of the surfaces play a very important role in the surface wetting phenomenon. The van der Waals interactions contribute the most to the surface free energy of the models studied here, even for the systems with strong electrostatic interactions. No obvious orientation and/or elongation effect of polymer chain on the shape of droplets is seen in this study.

\section{ACKNOWLEDGMENTS}

The authors wish to thank Dr. R. M. Thompson and Dr. B. Hehn of W. L. Gore \& Associates, Inc. and Professor W. A. Goddard III of Caltech for many helpful discussions and suggestions. This research is partially supported by W. L. Gore \& Associates, Inc.

\section{APPENDIX}

The equation given by Hautman and Klein to calculate contact angle is

$$
\left\langle z_{\text {c.m. }}\right\rangle=(2)^{-4 / 3} R_{0}\left(\frac{1-\cos \theta}{2+\cos \theta}\right\rangle^{1 / 3} \frac{3+\cos \theta}{2+\cos \theta},
$$

where $z_{\text {c.m. }}$ is the average height of the center of mass of water droplet, $R_{0}$ is the radius of free spherical drop of $N$ water molecules, and $\theta$ is the contact angle. By definition the center of mass of an intersected sphere can be calculated from:

$$
z_{\text {c.m. }}=\frac{\int_{0}^{h} z S(z) d z}{\int_{0}^{h} S(z) d z}
$$

where $S(z)=\pi r_{z}^{2}$ is the cross section at height $z$ with $r_{z}^{2}=R^{2}-(R-h+z)^{2}$. Other parameters are defined in Fig. 2. By integrating Eq. (A2) and replacing $h / R$ with $(1-\cos \theta)$ the $z_{\text {c.m. }}$ can be expressed as

$$
z_{\text {c.m. }}=\frac{R}{4} \frac{(3+\cos \theta)(1-\cos \theta)}{2+\cos \theta} .
$$

The $R$ and $R_{0}$ can be related through the volume of the droplet:

$$
V=\pi h^{2}\left(R-\frac{h}{3}\right)=\frac{4}{3} \pi R_{0}^{3},
$$

which gives

$$
R=\frac{(2)^{2 / 3} R_{0}}{(1-\cos \theta)^{2 / 3}(2+\cos \theta)^{1 / 3}} .
$$

Combining Eqs. (A3) and (A5) leads to Eq. (A1), a fourth order equation. The $R_{0}=\left(3 N / 4 \pi \rho_{0}\right)^{1 / 3}$ can be obtained by assuming the droplet with constant and uniform density $\rho_{0}=0.033 \AA^{-3}$. Thus, the contact angle becomes a function of a single parameter $z_{\text {c.m. }}$. 
In principle our approach is equivalent to Hautman and Klein's since identical geometric relationships are used. However, the direct computation of $V$ removes the assumption about $R_{0}$ and the calculation of interfacial area applies to any surface geometries. Therefore, the approach presented in this paper represents an improvement.

${ }^{1}$ Contact Angle, Wettability and Adhesion, Adv. Chem. Series Vol. 43, edited by F. M. Fowkes (American Chemical Society, Washington, DC, 1964).

${ }^{2}$ B. W. Cherry, Polymer Surfaces (Cambridge University, Cambridge, UK, 1981).

${ }^{3} \mathrm{~S}$. Wu, Polymer Interface and Adhesion (Marcel Dekker, New York, 1982).

${ }^{4}$ P. G. de Gennes, Rev. Mod. Phys. 57, 827 (1985).

${ }^{5}$ J. Hautman and M. Klein, Phys. Rev. Lett. 67, 1763 (1991).

${ }^{6}$ W. Mar and M. Klein, J. Phys. Condensed Matter 6, A381 (1994).

${ }^{7}$ H. Tadokoro, Structure of Crystalline Polymers (Wiley, New York, 1979), and references therein.

${ }^{8}$ I. C. Sanchez, Physics of Polymer Surfaces and Interfaces (ButterworthHeinemann, Boston, 1992).
${ }^{9}$ S. L. Mayo, B. D. Olafson, and W. A. Goddard III, J. Phys. Chem. 94, 8897 (1990)

${ }^{10}$ J. Gasteiger and M. Marsili, Tetrahedron 36, 3219 (1980).

${ }^{11}$ H. J. Berendsen, J. R. Grigera, and T. P. Straatsma, J. Phys. Chem. 91, 6269 (1987)

${ }^{12}$ H. J. Berendsen, J. P. M. Postma, W. F. van Gunsteren, and J. Hermans, in Intermolecular Forces, edited by B. Pullman (Reidel, Dordrecht, Holland, 1981).

${ }^{13}$ W. L. Jorgensen, J. Chandrasekhar, J. D. Madura, R. W. Impey, and M. L. Klein, J. Chem. Phys. 79, 926 (1983).

${ }^{14}$ J. J. de Pablo and J. M. Prausnitz, J. Chem. Phys. 93, 7355 (1990).

${ }^{15}$ Y. Guissani and B. Guillot, J. Chem. Phys. 98, 8221 (1993).

${ }^{16}$ J. Alejandre and D. J. Tildesley, J. Chem. Phys. 102, 4574 (1995)

${ }^{17}$ A. K. Rappe and W. A. Goddard III, J. Phys. Chem. 95, 3358 (1991).

${ }^{18}$ S. Nose, Mol. Phys. 52, 255 (1984); J. Chem. Phys. 81, 511 (1984).

${ }^{19}$ M. P. Allen and D. J. Tildesley, Computer Simulation of Liquids (Clarendon, Oxford, UK, 1987), and references therein.

${ }^{20}$ D. K. Owens and R. C. Wendy, J. Appl. Polym. Sci. 13, 1741 (1969), and references therein.

${ }^{21}$ W. A. Zisman, in Contact Angle, Wettability and Adhesion, Adv. Chem. Series Vol. 43, edited by F. M. Fowkes (Am. Chem. Soc., Washington, DC, 1964) 